\title{
The Influence of Different Geometries for a Vaned Diffuser on the Pressure Distribution in a Centrifugal Compressor *
}

\author{
Ingolf Teipel and Alexander Wiedermann \\ Institute for Mechanics, University of Hannover, Hannover, Germany
}

\begin{abstract}
$\star$ Presented at the 1984 ASME Meeting in Amsterdam
Abstract

In this paper the transonic flow field in a vaned diffuser of a centrifugal compressor has been studied. Different geometries of the diffuser blades have been considered. Particularly with respect to the pressure field it is important to understand their influence on the development of the shock waves. For this problem a centrifugal compressor with high pressure ratio per stage has been examined. It is assumed that the impeller speed is so large that the flow may become transonic at the impeller exit. To calculate flows with shocks a time-marching method is used for solving the two-dimensional Euler equations in a conservative form. A MacCormack's second order difference scheme is applied. There are two important points which have been studied in this paper. Firstly the geometry of the blades as far as the thickness distribution is concerned is kept unchanged and only the stagger angle has been altered. The performance maps of these diffusers and their effect on the whole machine will be discussed. Secondly the results which have been obtained by using vanes with different thickness distributions will be presented. Mean values of the quantities used for the performance map will be shown. Wall pressure distributions as well as fields of isobars and of constant Mach numbers will be demonstrated to provide a better understanding of the pressure recovery in centrifugal compressor diffusers.
\end{abstract}

\section{Nomenclature}

$c_{p}$

d

$$
\text { F }
$$

G

$\mathrm{H}$

$$
\mathrm{h}_{\mathrm{t}}
$$

$$
\mathrm{M}
$$

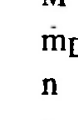

\section{n}

\section{$\mathrm{p}$}

$$
\begin{aligned}
& \mathrm{p} \\
& \mathrm{pt}
\end{aligned}
$$$$
\text { t }
$$

pressure recovery coefficient maximum blade thickness, $\mathrm{mm}$ matrix, def. in Eq. (1) matrix, def. in Eq. (1) matrix, def. in Eq. (1)

$$
\text { total enthalpy, } \mathrm{J} / \mathrm{kg}
$$$$
\text { local Mach number }
$$$$
\text { dimensionless mass flux, def. in Eq. (5) }
$$$$
\text { rotating speed, } \min ^{-1}
$$$$
\text { radius, } m
$$$$
\text { length of the chord of a blade, } m
$$$$
\text { pressure, bar }
$$$$
\text { total pressure, bar }
$$$$
\text { time, } s
$$$$
\text { matrix, def. in Eq. (1) }
$$$$
\text { radial velocity component, } \mathrm{m} / \mathrm{s}
$$$$
\text { tangential velocity component, } \mathrm{m} / \mathrm{s}
$$

$$
\text { angle }
$$$$
\text { relation of specific heats }
$$$$
\text { angle }
$$$$
\text { diffuser radius ratio, } \lambda=r / r_{\mathrm{IE}}
$$$$
\text { density, } \mathrm{kg} / \mathrm{m}^{3}
$$

\section{Indices}

\section{IE Impeller exit \\ DE Diffuser exit}

\section{Introduction}

During the last few years the flow pattern in centrifugal compressors has been examined experimentally as well as theoretically under various aspects. In most cases emphasis was put on the rotating parts of the machine and in a great number of research reports three-dimensional effects of the flow field in centrifugal compressor rotors have been studied $/ 1,2,3 /$. However, the development to high pressure ratios per stage has forced the requirement for a new design of the diffusers. To maintain a reasonable size of a unit vaneless diffusers no longer have been used. In order to optimize the efficiency of the compressor the blades of vaned diffusers are mostly formed with respect to aerodynamic rules.

A more or less strong interaction between the impeller and the diffuser is responsible for the complicated flow pattern, especially in the vaneless region between rotor and stator. It is not sufficient 
to consider only the impeller flow. As a main consequence of the blockage because of the blades in the diffuser the maximum mass flux will be reduced in comparison to that of a compressor with a vaneless diffuser. This means that the performance chart becomes smaller. But on the other hand a better pressure recovery will be obtained by using vaned diffusers.

When considering high-loaded units supersonic regions might occur behind the throat of a diffuser channel if the choking line is reached. In such cases the compressor characteristic is a vertical line. On the other hand very high impeller speeds cause transonic flow conditions in the vaneless region between impeller exit and diffuser inlet, even at the design point. Oblique shock waves occur in front of the vanes. Therefore a numerical procedure has to be chosen which shall be able to calculate transonic flow regions including discontinuities. These requirements have to be completed by a time-marching method. When retaining time derivatives the fundamental equations remain of a hyperbolic type both for subsonic and supersonic regions.

A time-marching scheme for calculating twodimensional inviscid flow fields in vaned diffusers was recently used $/ 4,5 /$. Comparisons of calculated and measured flow quantities have been made and the agreement has been found to be reasonably good, as far as the pressure and the Mach number distributions are concerned. Because of the assumption of inviscid flow no correct results of the efficiency can be obtained. In this paper further results obtained by the time-marching method will be presented. The purpose here is to show the influence of different geometries of the blades on the pressure field.

\section{Description of the Method}

In order to capture shock waves a conservative formulation of the governing equations has been chosen. In a cylindrical coordinate system it can be expressed in the matrix form:

$$
\frac{\partial \mathrm{U}}{\partial \mathrm{t}}+\frac{\partial \mathrm{F}}{\partial \mathrm{r}}+\frac{\partial \mathrm{r}}{\partial \theta}+\mathrm{H}=0
$$

with the matrices

$$
\begin{aligned}
& \mathrm{U}=\left[\begin{array}{ll}
\mathrm{r} & \rho \\
\rho & \mathrm{u}_{\mathrm{r}} \\
\rho & \mathrm{u}_{\theta}
\end{array}\right], \quad \mathrm{F}=\left[\begin{array}{lll}
\mathrm{r} & \rho & \mathrm{u}_{\mathrm{r}} \\
\rho & \mathrm{u}_{\mathrm{r}}^{\hat{1}} & +\mathrm{p} \\
\rho & \mathrm{u}_{\mathrm{r}} & \mathrm{u}_{\theta}
\end{array}\right] \text {, } \\
& \mathrm{G}=\left[\begin{array}{lll}
\rho & \mathrm{u}_{\theta} \\
\rho & \mathrm{u}_{\mathrm{r}} & \mathrm{u}_{\theta} / \mathrm{r} \\
\left(\rho \mathrm{u}_{\theta}^{2}+\mathrm{p}\right) / \mathrm{r}
\end{array}\right], \quad \mathrm{H}=\left[\begin{array}{c}
0 \\
\rho\left(\mathrm{u}_{\mathrm{r}}^{2}-\mathrm{u}_{\theta}^{2}\right) / \mathrm{r} \\
2 \rho \mathrm{u}_{\mathrm{r}} \mathrm{u}_{\theta} / \mathrm{r}
\end{array}\right]
\end{aligned}
$$

The different rows of the matrices denote the continuity equation and the radial and tangential component of the momentum equation. For jsoenergetic flow the energy equation can be replaced by the algebraic expression:

$$
\mathrm{h}_{\mathrm{t}}=\frac{\ddot{i}}{\gamma-1} \frac{\mathrm{p}}{\rho}+\frac{1}{2}\left(\mathrm{u}_{\mathrm{r}}^{2}+\mathrm{u}_{\theta}^{2}\right)=\text { const. }
$$

To fulfill the boundary conditions along the solid walls the physical plane is mapped into a more regular computational domain such that the pressure and suction side of the blades become coordinate lines. A stretching of the grid system can be carried out by an analytical transformation. This may be important, if the gradients become strong. Eq. (1) is solved by an explicit MacCormack scheme using a predictor and a corrector step $/ 6 /$.

The inlet and the exit regions of the domain are treated with algorithms derived from characteristic theory. In the vaneless region periodic boundary conditions are applied in the tangential direction. Along the blades the streamline condition is used and the pressure is calculated by the momentum equation normal to the wall. The time step size is determined by the CFL-condition and the iteration procedure will be stopped if a certain convergence limit is reached. More details are outlined in $/ 7 /$.

\section{Different Diffuser Geometries}

All investigated geometries are variations of an original diffuser called type (a). The original diffuser is equipped with 19 blades. Comparisons of theoretical and measured data obtained with geometry of type (a) have already been presented in $/ 4 /$ and $/ 5 /$. The important parameters of the investigation are the stagger angle and the thickness distribution of the blades.

The stagger angle has been altered by rotating 
each of the blades around the leading edge. The diffuser inlet radius and the profile of the blades are kept constant. Secondly the thickness distribution of the blades has been changed. The original blade was thickened by adding a parabola, both to the suction and to the pressure side. All essential geometrical data are listed in Table 1 . The diffusers (b) and (c) are formed through variation of the stagger angle and the type(d)has been obtained by altering the thickness distribution.

TABLE 1

\begin{tabular}{lllll}
\hline geometry & a & b & c & d \\
impeller exit radius & $200 \mathrm{~mm}$ & $200 \mathrm{~mm}$ & $200 \mathrm{~mm}$ & $200 \mathrm{~mm}$ \\
diffuser inlet radius & $230 \mathrm{~mm}$ & $230 \mathrm{~mm}$ & $230 \mathrm{~mm}$ & $230 \mathrm{~mm}$ \\
diffuser outlet radius & $300 \mathrm{~mm}$ & $287 \mathrm{~mm}$ & $305 \mathrm{~mm}$ & $300 \mathrm{~mm}$ \\
relative thickness d/s & $3 \%$ & $3 \%$ & $3 \%$ & $4.5 \%$ \\
stagger angle & $7.5^{\circ}$ & $2.5^{\circ}$ & $9.5^{\circ}$ & $7.5^{\circ}$ \\
\hline
\end{tabular}

\section{Variation of the Stagger Angle}

Now the flow field in diffusers of type (b) and (c) are discussed. As can be seen from Table 1 type (b) has been given by rotating the blades at $\Delta \varphi=$ $-5^{\circ}$ against the radial direction. For the diffuser type (c) the value of $\Delta \varphi$ is $+2^{\circ}$. The different geometries can be seen directly in Fig. 5 and Fig. 6 . The original blades have been drawn with dotted lines. In all cases in the investigation the impeller speed was $14,600 \mathrm{~min}^{-1}$ and the Mach number at the diffuser inlet was about 0.9 .

Fig. 1 shows the calculated performance chart of the different diffuser types. The pressure recovery coefficient $c_{p}$ is defined by

$$
c_{p}=\frac{\bar{p}_{D E}-\bar{p}_{I E}}{p_{t}-\bar{p}_{I E}}
$$

where $\overline{\mathrm{p}}_{\mathrm{IE}}$ and $\overline{\mathrm{p}}_{\mathrm{DE}}$ are average pressure values at the impeller exit and the diffuser exit and $p_{t}$ is the total pressure. $c_{p}$ is plotted versus a nondimensional mass flux $\dot{m}_{D}$ defined by

$$
\dot{\mathrm{m}}_{\mathrm{D}}=\frac{1}{2 \pi \cdot \mathrm{r}_{\mathrm{IE}} \cdot \sqrt{\mathrm{p}_{\mathrm{t}} \rho_{\mathrm{t}}}} \int_{0}^{2 \pi} \rho \mathrm{u}_{\mathrm{r}} \mathrm{r} \mathrm{d} \theta
$$

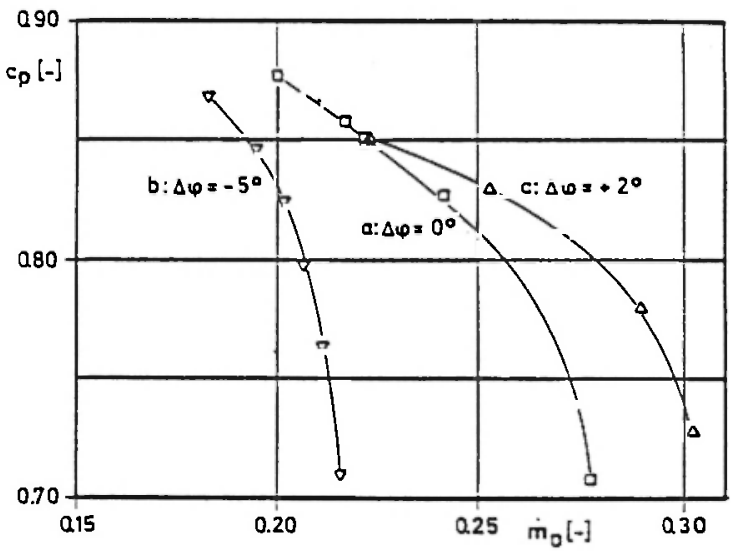

Fig. 1. Characteristics of diffusers with different stagger angles; $n=14,600 \mathrm{~min}^{-1}$.

For high-loaded machines the maximum mass flux is determined by the throat area of the diffuser channel. Therefore the surge line of the diffuser is identical to that of the whole compressor. The reduction of the stagger angle gives a smaller channel width and for example the mass flux of type(b) is about $20 \%$ smaller than in original case. As expected the geometry of type (c) yields a larger mass flux. The value is about $15 \%$. At maximum mass flux all diffuser characteristics end in vertical lines because the cascade is choked, i.e. sonic speed is reached in the throat.

The left limits of the different curves in Fig. 1 are determined by operating points where small oscillations of the flow variables cannot be damped out anymore. A further increase of the exit pressure leads to numerical instabilities. Here it has been found that there exists a correlation between the numerical stability of the inviscid procedure and the physical stability of the flow at the choking line. In both cases the angle of incidence of the blades is too large, such that strong gradients occur in front of the cascade while in the interior of the diffuser channel a very weak pressure rise is found.

Fig. 2 and Fig. 3 show the average flow angles at the diffuser inlet $\alpha_{I E}$ and at the diffuser exit $\alpha_{\mathrm{DE}}$. At small stagger angles (type $b$ ) there is only a small variation of $\alpha_{\mathrm{IE}}$ with respect to the exit pressure. With increasing $\Delta \varphi$ the flow reacts very sensitively to altered pressure conditions at the diffuser exit. Here at the outlet the flow angle $\alpha_{D E}$ is nearly constant for all operating points.

Detailed information about the pressure recovery in the diffuser will be given by the fields of isobars. 


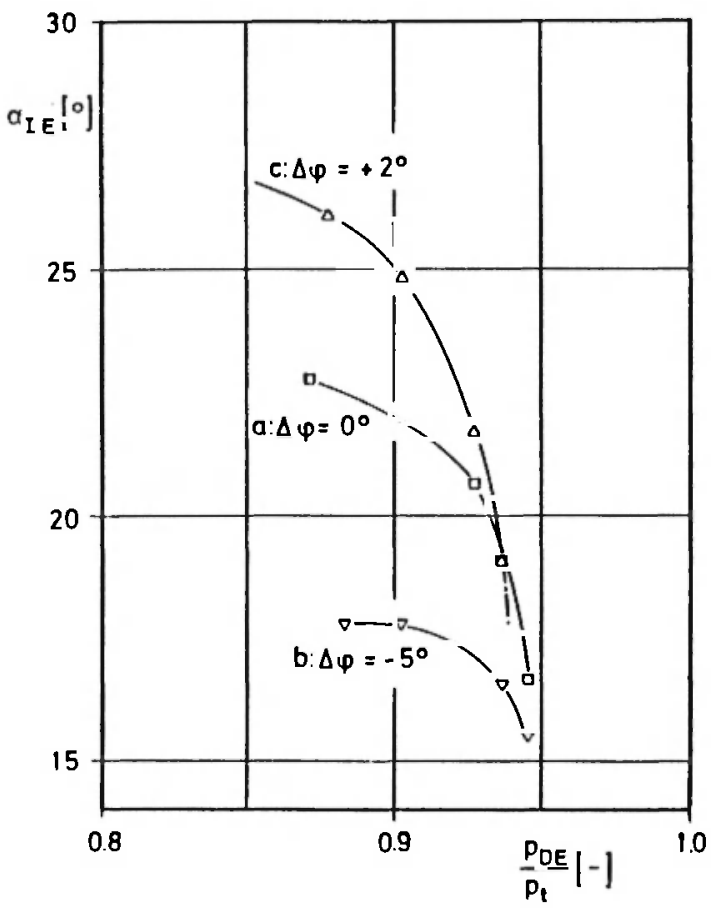

Fig. 2. Average outlet angle of the impeller at different operating points; $n=14,600 \mathrm{~min}^{-1}$.

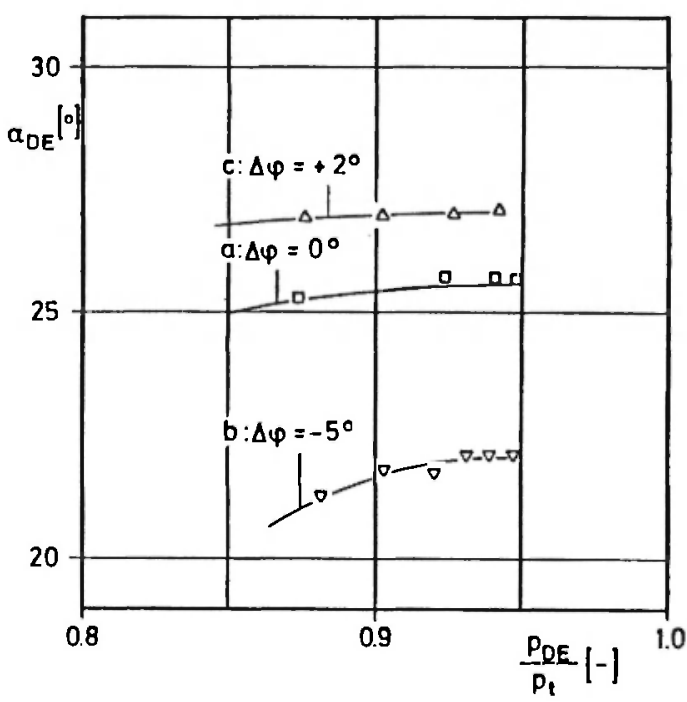

Fig. 3. Average outlet angle at the diffuser $\lambda=1.55 ; \mathrm{n}=14,600 \mathrm{~min}^{-1}$.

They are shown for the case when the cascade is nearly choked. Lines of constant static pressure divided by the total pressure are plotted in Figs. 4,5 and 6 . To compute the whole pressure field for every operating point a CPU-time of $5 \mathrm{~min}$. at a CDC Cyber 76 was needed. At the starting point

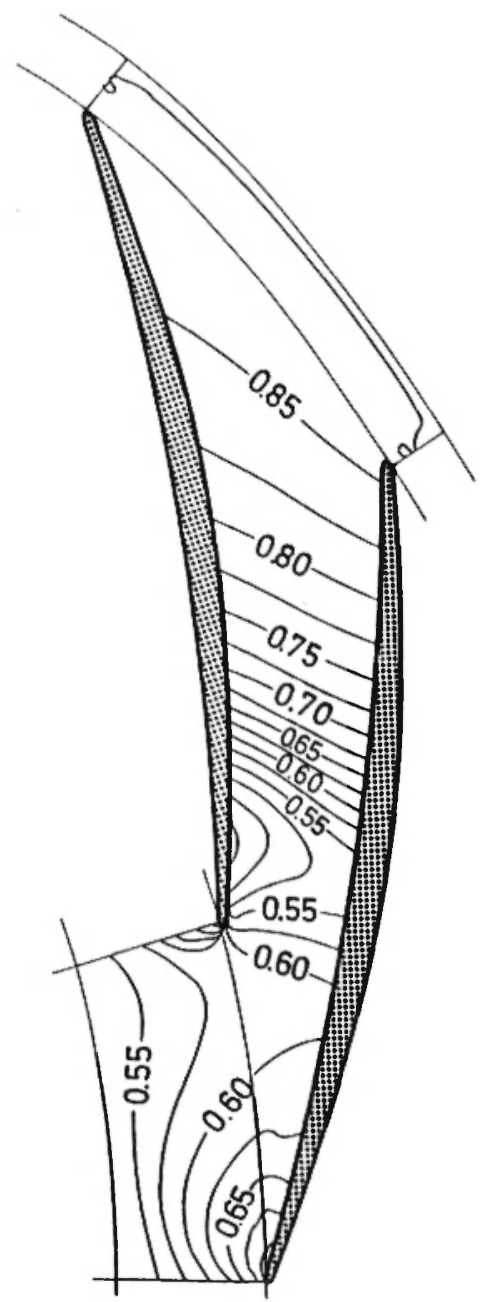

Fig. 4. Field of isobars $p / p_{t} ; n=14,600 \mathrm{~min}^{-1}$. Type (a)

of the calculation results of an one-dimensional theory have been used.

For type (a) and (b) the flow field is characterized by a supersonic regime in the region behind the throat. For air with $\gamma=1.4$ the critical pressure ratio is 0.528 . Weak shock waves can be found in the diffuser channel where many isobars are coming close together. In the case $c$ with $\Delta \varphi=$ $+2^{\circ}$ (Fig. 6) the sound speed is not yet reached at the throat. Only at the pressure side a small local supersonic region appears. Behind the impeller exit the isobars are of a wavy form. This effect can also be observed by experiments. For all three types the same pressure at the diffuser exit was assumed. 


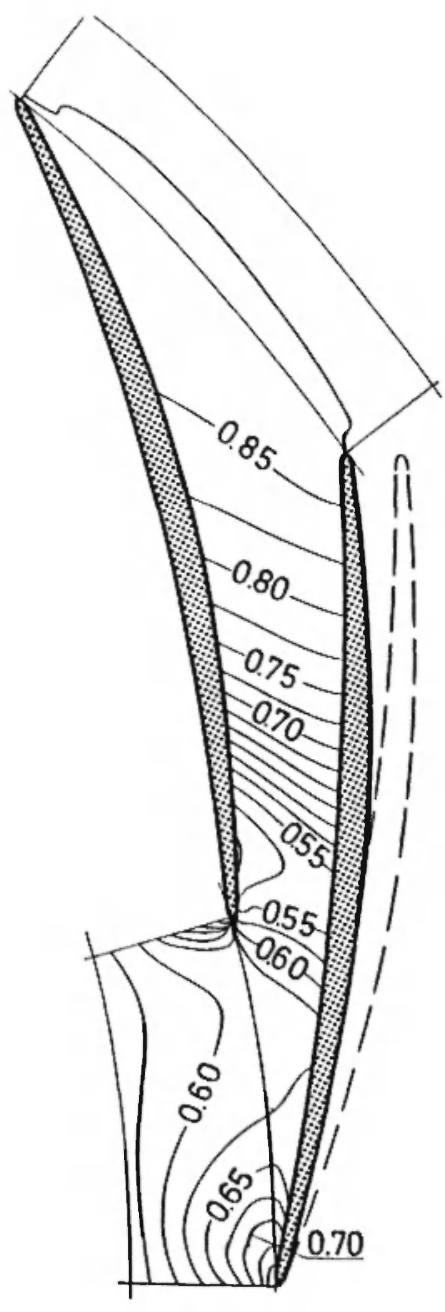

Fig. 5. Field of isobars $\mathrm{p} / \mathrm{p}_{\mathrm{t}} ; \mathrm{n}=14,600 \mathrm{~min}^{-1}$. Type (b)

\section{Variation of the Thickness Distribution}

Now the influence of different blade thickness distributions will be discussed. As shown in Table 1 the new relative maximum thickness of the blade is assumed to be $4.5 \%$. In Fig. 7 the characteristics of diffuser type (a) and type (d) are demonstrated. The blockage is now larger because of the thicker blades and the maximum flux becomes smaller. On the other hand the curves for (a) and (d) are very similar to each other in the region where the flow is choked. Basically thicker blades lead to a narrower performance chart and therefore the operation conditions of such compressors are more limited.

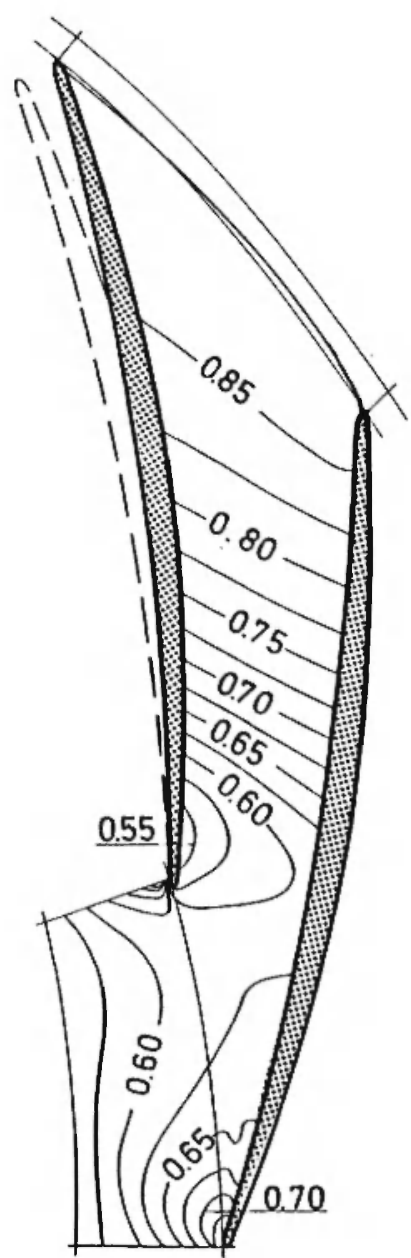

Fig. 6. Field of isobars $\mathrm{p} / \mathrm{p}_{\mathrm{t}} ; \mathrm{n}=14,600 \mathrm{~min}^{-1}$. Type (c)

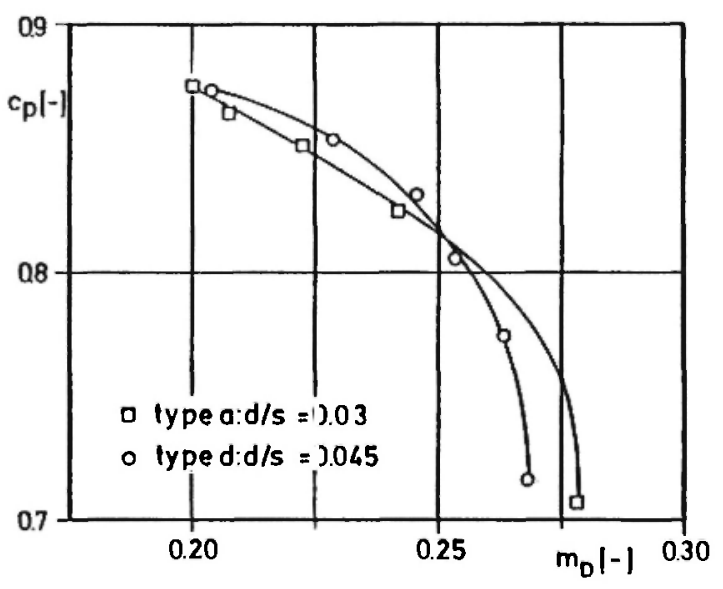

Fig. 7. Characteristics of type (a) and type (d). $\mathrm{n}=14,600 \mathrm{~min}^{-1}$. 
Wall pressure distributions give a direct information about the occurrence of steep gradients which might cause separation. In Fig. 8 and 9 they are

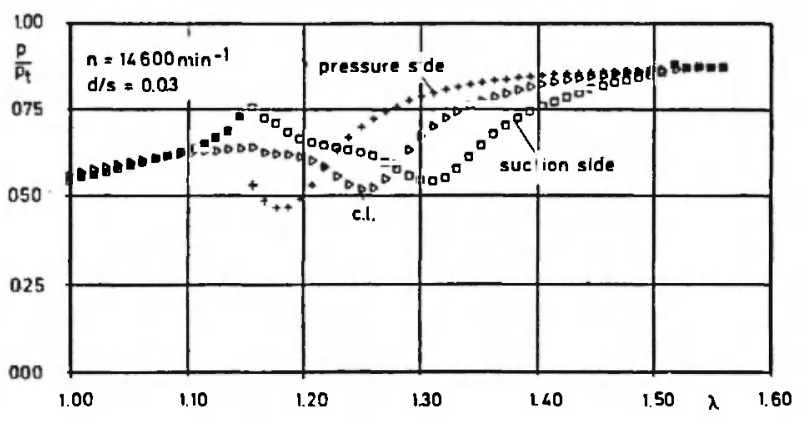

Fig. 8. Wall pressure, type (a).

c.L.: centre line between two neighbouring blades,

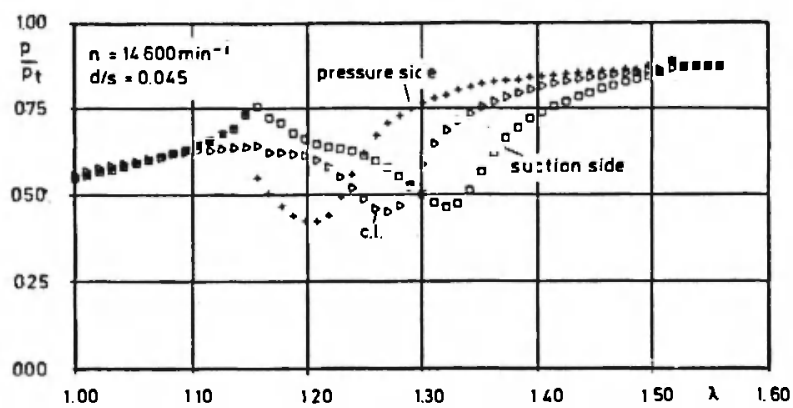

Fig. 9. Wall pressure, type (d).

shown for type (a) and type (d) and as operating point one has chosen a point on the choking line. $\lambda$ is the original radius divided by the impeller exit radius. The vaned region begins at $\lambda=1.15$ and ends at $\lambda=1.50$. In both cases the diffuser exit pressure was $88 \%$ of the total pressure. A faster acceleration of the flow can be observed for the thicker blades in the first part of the channel. It ends with a shock wave.

This can be seen very clearly in the field of isobars, Fig. 10. In the middle of the channel the isobars come together very closely. Here a shock wave will be obtained. The pressure distribution in the vaneless region of the diffuser has not been changed much in comparison to the original type (a).

\section{Concluding Remarks}

In this paper results obtained by an inviscid

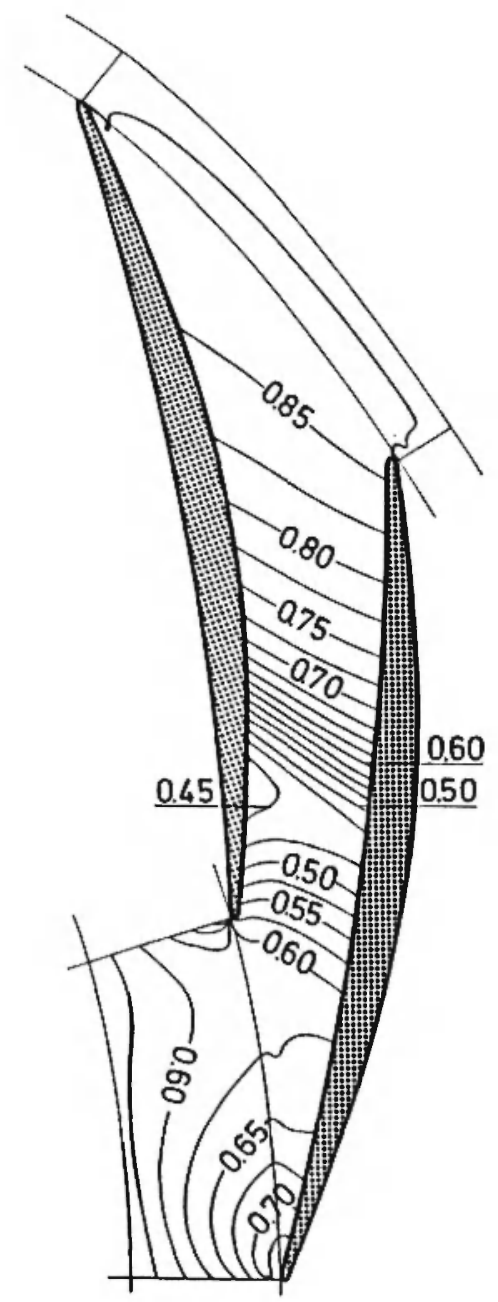

Fig. 10. Field of isobars $\mathrm{p} / \mathrm{p}_{\mathrm{t}} ; \mathrm{n}_{\text {red }}=14,600 \mathrm{~min}^{-1}$ Type (d).

theory for the flow field in a vaned diffuser have been discussed. The influence of various geometrical parameters have been studied. One can get reasonable information about the performance chart as well as details of the flow field. As a time-marching method has been used, pressure distributions with supersonic regimes and even with shocks could easily be obtained. However until now there is no possibility to calculate the efficiency because no loss model was formulated. An attempt to get information about losses by determining the boundary layer growth using integral methods did not give correct results. In cases where the flow angle at the diffuser inlet was quite different from the stagger angle, separation occurred very early and therefore boundary layer calculations could not be extended to the end of the blade. Nevertheless the 
inviscid theory provides a good understanding of some important flow phenomena in radial diffusers. The authors do not know of any existing experimental data relating to the present work.

\section{References}

1. J.H.G. Howard and C. Osborne, "A Centrifugal Compressor Flow Analysis Employing a Jet-Wake Passage Flow Model", Journal of Fluids Engineering, Vol. 99, No. $1,1977$.

2. Y. Krimermann and D. Adler, "The Complete ThreeDimensional Calculation of the Compressible Flow Field in Turbo-Impellers", Journal of Mechanical Engineering Science, Vol. 20, No. 3, 1978.
3. D.A. Fraser, J.H.G. Howard and W.C. Lennox, "A ThreeDimensional Turbulent Flow Analysis Method for the Rotating Channels of a Centrifugal Turbomachine", ASME-Paper 82-GT-221, 1982.

4. H.O. Jeske and I. Teipel, "Die Stroemung in Diffusoren fuer Radialverdichter an der Schluckgrenze", Forschung auf dem Ingenieurwesen, Vol. 47, No. 6, 1981.

5. H.O. Jeske and I. Teipel, "A Theoretical Investigation of Transonic Flow in Radial Compressor Diffusers", ASME-Paper 82-GT-227, 1982.

6. R.W. MaoCormack, "The Effect of Viscosity in Hypervelocity Impact Cratering", AIAA-Paper, 69-354, 1969.

7. H.O. Jeske, "Die transsonische Stroemung in beschaufelten Diffusoren von Radialverdichtern", Ph.D. Thesis, Hannover, 1980. 
Brought to you by | University of Arizona Authenticated 